\title{
Interacción enfermera-paciente y su repercusión en el cuidado hospitalario. Parte I
}

\section{Nurse-patient interaction and its repercussion to hospital care. Part 1}

Mtra. en S.P. María Cristina Müggenburg Rodríguez Vigil•

Dra. en Psic. Angélica Riveros-Rosas.•

\section{Resumen}

Se realizó una amplia búsqueda en diversas bases de datos a partir de los descriptores: interacción o comunicación enfermera-paciente, satisfacción por el cuidado de enfermería y entrenamientos en técnicas de comunicación, con el fin de profundizar en el tema de la interacción enfermera-paciente por la importancia que esta tiene en el ejercicio de la práctica profesional de las enfermeras.

Se establecen tres ejes de estudio: Interacción o comunicación enfermera-paciente, calidad y satisfacción derivada del cuidado, y efectividad de los entrenamientos en habilidades para la comunicación.

La revisión permite identificar que este tema ha sido analizado desde hace mucho tiempo y en diferentes países, los estudios se han relacionado con la calidad de los servicios de enfermería y han motivado el planteamiento de estrategias educativas para desarrollar y mejorar la interacción enfermera-paciente como actitud profesional esencial.

Los estudios realizados ofrecen elementos de apoyo para el desarrollo de investigaciones en esta área, pero en algunos casos muestran limitaciones o carencias, así como resultados contradictorios que pueden ser tomadas en cuenta por las personas interesadas en el diseño de estudios relacionados con el tema.

- Profesora de Carrera ENEO-UNAM, alumna del Doctorado en Psicología de la UNAM

•• Profesora de Carrera UNAM, Tutor del Programa de Doctorado en Psicología UNAM.

Correspondencia: cmuggenb@yahoo.com.mx

FeCha DE ReCibido: $1^{\circ}$. DE OCTUBRe 2011

Fecha de Enviado: 5 DE NOVIEMBre 2011

Fecha de ACeptado: 11 DE Diciembre 2011 


Key
Words:
nurse-patient
relations; nurse-patient
communication;
healthcare
communication and
satisfaction;
nurse-patient
communication
training.

Abstract

In order to deepen into the highly important topic nurse-patient interaction for the professional practice of nurses, a database search using the descriptors: nursepatient interaction/communication, nurse healthcare satisfaction, and communication skills training, was conducted.

As a result, three study axes were established: nurse-patient interaction/communication, care-derived quality and satisfaction, and communication skills training effectiveness.

This review reveals that this topic has been extensively analyzed throughout time and among different countries. These studies have been related to quality in nursing services, and have motivated the design of strategies to develop and improve the nurse-patient interaction as an essential professional attitude.

Furthermore, although these studies offer elements to support the research development in this area, some of them have shown limitations o deficiencies, as well as contradictory results, which should be considered by researchers interested in the design of studies related to this topic.

\section{INTRODUCCIÓN.}

La interacción o comunicación enfermera-paciente ocupa un lugar prioritario en el ejercicio de la práctica profesional de las enfermeras, ya que es el eje articulador de los cuidados, tanto asistenciales, como tecnológicos, dirigidos a la oferta del cuidado de enfermería de calidad.

Bajo esta premisa se realizó una amplia búsqueda a partir de los descriptores: relaciones enfermera-paciente, comunicación enfermera-paciente, cuidado de enfermería y satisfacción del paciente, entrenamiento para la comunicación en enfermeras, efectos de los entrenamientos en comunicación enfermera-paciente. Se consultaron bases como: Medline, Base de datos Cochrane y Cuiden de Enfermería; la localización de los primeros artículos que se consideraron de valor para la orientación de la búsqueda planeada, motivaron a la recuperación de artículos afines, referidos en estos, lo que permitió la ampliación y profundización en el tema. A través de esta exploración se ha podido identificar desde cuándo se ha estudiado este fenómeno, quiénes lo han investigado, con qué propósito, con qué metodologías, qué hallazgos han encontrado, qué similitudes se han establecido, qué diferencias se han hallado, qué planteamientos se han confirmado y qué limitaciones se han reportado.

Se establecen tres ejes de estudio, los cuales por razones de espacio se dividirán en dos partes; en esta primera parte se abordan los dos primeros ejes y en una segunda el tercero,
- Interacción o comunicación enfermera-paciente.

- Calidad y satisfacción derivada del cuidado.

- Efectividad de los entrenamientos en habilidades para la comunicación.

\section{Interacción o comunicación enfermera-paciente.}

Ha sido analizada a través de revisiones sistemáticas como la de Bengoechea, ${ }^{1}$ quien recogió los 20 estudios más significativos sobre la comunicación enfermera-paciente en áreas de cuidados críticos, la mayoría realizados con enfoque de tipo cualitativo, teniendo como criterio el análisis de las características de la forma de comunicación empleada por la enfermera en su interacción con el paciente, dichos 
estudios fueron realizados entre 1980 y 1999.

Entre los hallazgos identificados se encontraron los siguientes: Stovsky B (citado por Bengoechea) señala que la enfermera emplea el lenguaje verbal técnico, establece contactos físicos breves y no planificados basados en el cuidado físico y tecnológico sin considerar las emociones y reacciones de los pacientes, ${ }^{1}$ Salyer J. (citado por Bengoechea) observó pocas enfermeras facilitadoras de las relaciones, aunque esta situación se atenuó cuando las enfermeras eran expertas, mientras que las novatas se centraron en la tecnología buscando la seguridad del paciente, las enfermeras refirieron estrés ante las necesidades emocionales del enfermo, miedo ante las situaciones críticas, así como carencia en habilidades para la comunicación, situación que las centraba en la aplicación de cuidados técnico profesionales. ${ }^{1}$ También se identificaron obstáculos como: carga excesiva de trabajo y falta de habilidad en el manejo de las relaciones terapéuticas. En el caso de los estudios de tipo cualitativo, se concedió mayor énfasis a la observación del contacto visual, de las posturas relajadas y del tono de voz.

Frich $^{2}$ también llevó a cabo una revisión de la literatura que comprendió el periodo de 1993 a 2003 e identificó algunos resultados de las intervenciones de enfermería en adultos mayores con enfermedades crónicas cuyos padecimientos no estuvieran en etapas muy avanzadas, entre los hallazgos destacan: las intervenciones mejoraron los resultados clínicos como discapacidad, presión arterial, glucosa en sangre y síntomas de depresión, disminuyeron costos a partir de la reducción de días estancia o aumentaron algunos resultados del paciente como, la satisfacción personal (Campbell EM. Citado por Frich); el autoreporte de adherencia al tratamiento y la autoeficacia (Piette JD. Citado por Frich); a excepción del caso de algunos autores (Boehm S. Citado por Frich), quienes no encontraron diferencia en el mejoramiento de los resultados clínicos pero lo atribuyeron a la falta de discriminación de variables como edad, género y situación laboral; otros (Kirkman MS. Citado por Frich) hallaron poca mejoría en los autoreportes de los pacientes y poco efecto en la satisfacción referida por ellos mismos, explicando esto por la carencia de intervenciones multidisciplinarias. Entre las recomendaciones generales emitidas por el autor, se mencionó la necesidad de hacer más explícitas las intervenciones realizadas por el personal y estar atentos a los antecedentes educativos de los profesionales involucrados en éstas.

Finke, Light y Kitko $^{3}$ hicieron una revisión de artículos desde 1990 hasta 2007, estableciendo como criterio de búsqueda la comunicación de enfermeras y pacientes con necesidades complejas de comunicación, identificando barreras y apoyos para la comunicación efectiva; Localizaron 23 artículos, de los cuales se eliminaron 11 porque no señalaron criterios de inclusión; el análisis de los 12 restantes correspondió a ocho publicaciones de tipo cualitativo, tres desarrollados con enfoque cuantitativo y uno mixto. Entre los hallazgos identificados se encontró que la baja comunicación afecta la recuperación y consecuentemente a los días de estancia, además los pacientes reportaron frustración por las barreras en la comunicación.

Tay y Hegney ${ }^{4}$ seleccionaron 8 artículos relacionados con factores que afectan la comunicación en pacientes con Cáncer, desarrollados entre los años 2009 y 2010, de los cuales tres correspondieron al enfoque cuantitativo y cinco al cualitativo. Los pacientes fueron estudiados con el apoyo de videograbaciones y autoreportes, se evaluaron aspectos como la depresión, la ansiedad y la satisfacción con la comunicación, entre los hallazgos principales se señaló que las características de las enfermeras son factores clave para la comunicación.

En este mismo eje de estudio se encontraron diversas investigaciones de campo, ${ }^{5-7}$ porque diseñaron instrumentos para la medición de la interacción enfermera-paciente y demostraron su validez y confiabilidad; fundamentados en la filosofía de Watson (citado por Marriner y Raile $)^{8}$, midieron conductas interpersonales asociadas al cuidado (caring), Cossette y colaboradores ${ }^{5,6}$ con aplicaciones llevadas a cabo en estudiantes de enfermería, y Duffy y colaboradores ${ }^{7}$ lo midieron en situaciones reales con pacientes.

En el primer artículo de Cossette y colaboradores ${ }^{5}$ se describió el desarrollo de una versión corta de la escala CNPI (escala de interacción enfermera paciente, por sus siglas en inglés) con 23 reactivos que integran 4 dominios: cuidado clínico, con nueve reactivos; cuidado relacional con 7; cuidado humanístico con 4 y cuidado para el confort con 3; fue aplicada a 377 estudiantes de enfermería y se encontró que el cuidado clínico se identifica como el factor más tangi- 
ble en el trabajo hospitalario de las enfermeras. Esta escala tuvo como antecedente el estudio de diversos instrumentos (Larson PJ., así como Stanfield $\mathrm{MH}$. $)^{5}$ que retoman aspectos psicológicos del cuidado, fundamentados en Watson. ${ }^{8,9}$

En el segundo artículo, Cossette y colaboradores ${ }^{6}$ se enfocaron en la validación de constructo de las cuatro dimensiones de la escala, el cuidado clínico comprendió cuidados técnico profesionales; el cuidado relacional se concentra en la ayuda para explorar la importancia de la vida y el significado de la resolución eficiente de los problemas; el cuidado humanístico considera al paciente como individuo completo ofreciendo un apoyo que va más allá del padecimiento que sufre y el cuidado para el confort se relaciona con el respeto, la privacidad y la resolución de necesidades básicas. A partir de la aplicación del instrumento a 531 estudiantes de enfermería se llevó a cabo una validación de constructo por medio del análisis factorial, mostrando que la escala corta refleja los 4 factores propuestos; se encontró relación entre el factor humanístico y el relacional ( $\mathrm{r}$ $=.73$ ), el humanístico con el confort $(\mathrm{r}=.78)$ y el clínico con el confort $(\mathrm{r}=.83)$. Los autores hacen énfasis en la necesidad de estudiar y aplicar este tipo de escalas validadas con el fin de demostrar el impacto del cuidado, considerando no solamente los cuidados técnico-profesionales.

La aportación de Duffy y colaboradores, ${ }^{7,10}$ en cuanto al diseño y validación de instrumentos, se destaca en el Caring Assessment Tool (CAT, por sus siglas en inglés), diseñado para medir la percepción del cuidado desde el punto de vista del paciente.
El CAT fue inicialmente desarrollado en 1990 con el enfoque de la Teoría del Cuidado Humano de Watson, aplicando una escala tipo Likert usada para medir la experiencia de los pacientes durante su hospitalización, los reactivos fueron calificados de 1 a 5 , con el valor nunca en el primer caso y con el valor siempre en el último.

Se contó con un banco de 130 reactivos surgidos de la Teoría de Watson $^{9,11,}$ y un panel de 8 expertos que estudiaron el contenido y la validez de facie; los panelistas fueron instruidos hacia tres aspectos: a) pertinencia de cada reactivo como una conducta de cuidado de enfermería b) que cada reactivo representara algún factor relacionado con la teoría del cuidado y c) claridad del reactivo. Cada reactivo fue valorado como: 1 , de muy bajo cuidado o 5 , de muy alto cuidado. Los reactivos de 3.5 o más fueron retenidos, quedando 100; para los cambios de palabras se les pidió a los panelistas que se apegaran a los factores de la Teoría, siguiendo esta recomendación hicieron una segunda revisión en cuanto al contenido. Se midió consistencia interna seleccionando 86 pacientes hospitalizados en un servicio de médico quirúrgico y el alfa de Cronbach fue de .97; la redacción de los reactivos fue revisada en el año 2000 para capturar la realidad de los resultados y la consistencia interna permaneció alta, así como la validez de facie y de contenido, sin embargo empíricamente el instrumento era demasiado largo y cansaba a los pacientes por lo que se buscó un instrumento más corto. Se hizo un análisis en 557 pacientes, aplicando una rotación de varimax permaneciendo 64 reactivos, luego se aplicó un análisis de factores con rotación ortogonal. Al final quedaron 36 reactivos que valoraban ocho factores del cuidado. El instrumento con 36 reactivos fue probado en 365 pacientes para conocer su confiabilidad y el alfa de Cronbach fue de .96 , validando la consistencia interna del instrumento corto.

Dicho instrumento quedó constituido con ocho 8 factores o componentes del cuidado: 1) resolución mutua de problemas (siente que la enfermera le ayuda a resolver sus problemas); 2) atención al consuelo o reconfortamiento (disponibilidad e interés de la enfermera, da esperanzas, sensibilidad ante sus problemas); 3 ) respeto (acepta al paciente, le pone atención); 4) promoción del ánimo (demuestra empatía, calidez); 5) apreciación de significados únicos (percibe cómo los pacientes ven las cosas); 6) ambiente de sanación (tiene una visión holística); 7) adhesión a las necesidades (reconoce y apoya ante carencias); 8) necesidades básicas humanas (resuelve necesidades de carácter fisiológico). Y mostró una consistencia teórica y empírica creada por el panel de expertos, a la vez fue contrastado con teorías de enfermería existentes y diversos estudios realizados en la práctica. La consistencia teórica tuvo como referentes a: Leininger, ${ }^{12}$ Watson, ${ }^{9}$ Peplau, ${ }^{13}$ Nightingale, ${ }^{14}$ Roy, Swanson, King (citados por Marriner y Raile), ${ }^{8}$ y Henderson, ${ }^{15}$ quienes coinciden en la importancia de la interacción enfermera-paciente como elemento fundamental en el desempeño de la práctica profesional cotidiana. La evidencia empírica se fundamentó en Wolf y Cossette (citado por Duffy JR). ${ }^{10}$

Se considera que el CAT es un instrumento válido, confiable y fá- 
cilmente administrable, los valores pueden ir de 36 a 180; las calificaciones altas indican un grado alto de cuidado humanístico; el total del puntaje puede usarse para determinar el grado de percepción que tiene los pacientes acerca del cuidado de enfermería. También permite identificar elementos que pueden ser considerados para la formación de enfermeras, monitorear el mejoramiento en la práctica y sugerir cambios en ésta.

El trabajo publicado por Pytel, Fielden, Meyer y Albert ${ }^{16}$ también ofrece un aporte interesante en cuanto al diseño y aplicación de un instrumento relacionado con la interacción enfermera-paciente. Valoraron la percepción del paciente respecto a sus necesidades de comunicación, comparando la percepción de la enfermera con la del paciente, señalando importantes habilidades como el contacto visual, el tono de voz, el lenguaje adecuado al paciente, la información sobre terapéuticas, la actitud de escucha, el respeto, y la compasión, se observó coincidencia entre ambas percepciones con prioridad en los indicadores: ofrece confianza ante sus temores, ofrece confort y lo mantiene informado sobre pruebas y tratamientos; $y$ menor similitud entre la importancia del contacto ocular y el uso de voz templada, estos dos últimos con mayor reconocimiento por parte de la enfermera; la mayoría de estos indicadores también han sido reconocidos a través de estudios con enfoque cualitativo. ${ }^{17}$

Williams e Irurita ${ }^{18}$ describieron el contexto terapéutico de confort emocional identificando a través de un estudio cualitativo que explica la percepción terapéutica del

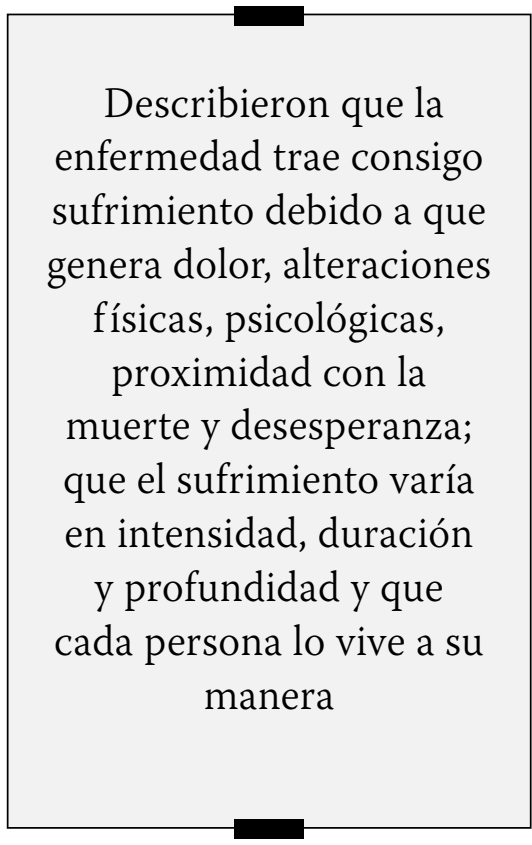

efecto de la interacción personal experimentada por los pacientes hospitalizados; años más tarde Williams y Kristianson ${ }^{19}$ desarrollaron un instrumento psicométrico que describe la experiencia emocional del paciente, lo aplicaron a 132 enfermos en busca de características de pacientes que requieren cuidado emocional, entre las que abordaron: edad, género, motivo de admisión, lugar de residencia, presencia de algún problema de comunicación, necesidad de ayuda en las necesidades diarias, disponibilidad de familiares y amigos para asistirlo. Realizaron validez de contenido y de facie, prueba piloto para analizar claridad, factibilidad y análisis factorial.

En este mismo eje de estudio, pero a través de metodologías con enfoque cualitativo otros autores $^{17,20-23}$ exploraron el significado del cuidado de enfermería. Describieron que la enfermedad trae consigo sufrimiento debido a que genera dolor, alteraciones físicas, psicológicas, proximidad con la muerte y desesperanza; que el su- frimiento varía en intensidad, duración y profundidad y que cada persona lo vive a su manera; por lo que la enfermera tendrá que acompañar, dar ánimo, esperanza y consuelo; y contrarrestar la soledad, la tristeza, la incertidumbre, la angustia y el dolor. Encontraron que el cuidado de enfermería depende de la presencia de las enfermeras al lado de los pacientes, quienes por lo menos esperan un saludo; que en muchas ocasiones el acercamiento con el enfermo se circunscribe a la realización de procedimientos dirigidos al cuidado físico y no a la asistencia emocional o psicológica. El paciente es considerado como una labor por cumplir, una tarea y un tratamiento por realizar, una enfermedad, un diagnóstico, un número de cama, razones por las cuales las enfermeras no ofrecen calor humano y afecto durante las interacciones del cuidado. Y que aún cuando la realización de procedimientos establecidos para la curación y recuperación de la salud de los pacientes pone en contacto directo a la enfermera con sus pacientes, no siempre observa ni interpreta los gestos, movimientos y posiciones con los cuales los pacientes comunican sus sentimientos, emociones y percepciones; además las dificultades para escuchar generan informaciones difusas incompletas o falsas que pueden conducir a decisiones o conclusiones erróneas.

Wysong y Diver ${ }^{24}$ también con el apoyo de metodologías cualitativas exploraron técnicas interpersonales, pensamiento crítico y habilidades técnicas identificadas por los pacientes. En cuanto a las técnicas interpersonales positivas los pacientes describieron a las enfermeras como: amigables, 
amables, afectuosas, compasivas, interesadas en su persona, confiables, alegres, les gusta su trabajo, sentido del humor, organizadas, buena memoria, buena apariencia, con buena escucha, confortadoras; en cuanto a técnicas negativas: tímidas, bruscas, rudas en sus respuestas, tensas, con actitud negativa, nerviosas, están de malas, pero deben dejar sus problemas en otro lado.

Respecto al pensamiento crítico afirmaron que toman decisiones clínicas rápidas y correctas, tienen habilidad para explicar signos y síntomas, complicaciones, intervenciones y uso de medicamentos. En cuanto a habilidades técnicas describieron aspectos positivos y fallas en la aplicación de inyecciones intravenosas y en el uso del equipo. Por último, concluyeron que la percepción del paciente sobre la enfermera tiene un fuerte componente para la construcción del significado del cuidado de enfermería. ${ }^{24}$

En síntesis, los estudios revisados sobre interacción o comunicación enfermera-paciente muestran como los profesionales de enfermería han estado preocupados, desde hace mucho tiempo y en diferentes espacios geográficos, en la relación interpersonal para el desempeño de su práctica.

Se encontró que hay predominio de investigaciones con enfoque cualitativo, en los cuales se observaron contactos físicos breves con el paciente, empleo de lenguaje técnico al hablar con él, actitudes bruscas, negativas o rudas al atenderlo; otros autores analizaron los beneficios de la interacción de la enfermera en cuanto a mejoría de datos clínicos e incremento de la satisfacción del paciente, con re- percusión en la recuperación o disminución de los días estancia hospitalaria; sin embargo estos últimos resultados no son consistentes.

En el ámbito cuantitativo el principal aporte se enfoca al diseño y validación de instrumentos que miden la interacción enfermera paciente, varios de estos tiene como sustento teórico a Watson, otros se abocaron a la percepción del paciente y de la enfermera en cuanto al uso de habilidades para la comunicación, destacando que la percepción del paciente tiene un fuerte componente para la construcción del significado del cuidado de enfermería.

Aún cuando hay una amplia producción en este eje de estudio, hay variaciones en los hallazgos, lo que deja abierto el campo a las enfermeras interesadas en el tema para seguir profundizando en el fenómeno de la comunicación o interacción.

\section{Calidad y satisfacción derivada del cuidado.}

Han sido estudiadas a través de diversas revisiones sistemáticas; ;5-27 $^{27}$ Kruijver y colaboradores ${ }^{25}$ en la revisión que realizaron de 1993 a 2004, observaron a través de 16 artículos elegidos que la identificación de problemas psicosociales en pacientes con cáncer, a través de listas de chequeo, era un factor importante para la individualización de estos, favoreciendo la resolución adecuada de los problemas y la calidad de atención recibida.

Charlton y colaboradores ${ }^{26}$ seleccionaron 26 artículos después de una revisión que efectuaron entre 1999 y 2005, al analizar los estilos de comunicación: biomédica y biopsicosocial, en el segundo caso observaron que los pacientes reportaban mayor satisfacción, más adherencia terapéutica y más bienestar cuando ésta era efectiva. Sin embargo, solamente 7 publicaciones cumplieron con los requisitos metodológicos relacionados con el tipo de diseño, aleatorización, criterios de inclusión, selección de la población y tamaño de muestra.

Por último, Tapia-Valero y colaboradores ${ }^{27}$ se interesaron en la búsqueda de evidencias para el mejoramiento de los resultados de salud y después de realizar una revisión de 626 artículos publicados en el lapso de 2000 a 2008, tomaron los 10 que ofrecen evidencia contundente, entre los factores condicionantes se identificó la atención individualizada, misma que se constituye como un factor fuertemente relacionado con la interacción personal.

La calidad del cuidado también ha sido estudiada por varios autores que relacionaron ésta con comportamientos propios de la interacción entre el personal de salud y los pacientes; en algunas investigaciones se correlacionaron positivamente: el comportamiento de la interacción enfermera-paciente con la calidad del cuidado, ${ }^{28}$ y $29 \mathrm{con}$ los días de estancia y la mejoría de componentes clínicos ${ }^{30}$ y con las necesidades del paciente, los buenos sentimientos y la empatía. ${ }^{31}$

Thomas y colaboradores, ${ }^{28}$ a través de un estudio realizado con 2078 pacientes de medicina interna y cirugía encontraron que la calidad del cuidado se relaciona con comportamientos favorables como el trato individualizado, las medidas de consuelo, la rapidez de respuesta ante las solicitudes del paciente, la supervisión periódica para la de- 
tección de necesidades, la respuesta a preguntas formuladas por el paciente, etc. Utilizaron la escala NSNS (Newcastle Satisfaction with Nursing Scales, por sus siglas en inglés), ésta mostró que los ancianos valoraron más la experiencia de la enfermera y que existía correlación entre la experiencia y la satisfacción del paciente cuando el enfermo reconoce a su enfermera. También se observó que la baja educación del paciente se relacionaba con la satisfacción positiva. Años más tarde, Torres-Contreras, ${ }^{29}$ aplicando la escala NSNS de Thomas identificó que la percepción positiva sobre el cuidado recibido de las enfermeras se relacionaba con la calidad del cuidado y con algunas variables sociodemográficas como el nivel de escolaridad del paciente. Confirmó que el trato individualizado era un factor importante en la valoración de la interacción enfermera-paciente.

Hojat, Gonnella, Nasca, Mangione, Vergare y Magee, ${ }^{32}$ utilizando la escala de empatía de Jefferson constituida por 20 reactivos de tipo Likert, encontraron que en 704 médicos encuestados, el incremento de la empatía del personal de salud se relacionó con la satisfacción del paciente y con mejoría clínica.

Miaofen y Li-Hua ${ }^{33}$ aplicaron a 1000 pacientes hospitalizados en Taiwan el PAHC (por sus siglas en inglés: Patient Assesment of Hospital Care), instrumento validado, integrado por siete dimensiones que exploran: valores, preferencias, necesidades, confort, apoyo emocional, miedo y ansiedad; y encontraron que la percepción de la calidad del cuidado que imparten las enfermeras se correlacionó con la satisfacción y el confort.
Mok y colaboradores ${ }^{31}$ a partir de un estudio fenomenológico exploraron las relaciones interpersonales entre los pacientes y las enfermeras utilizando variables como: contacto continuo y cercano, buenos sentimientos, empatía, respuesta a las necesidades del paciente, tiempo para escuchar al paciente entre otras, encontraron que la interacción favorable genera satisfacción en ambos y que las cualidades y técnicas desempeñadas por las enfermeras se relacionaban con el cuidado de excelencia.

Sierra, Cardona, Bernal y Forero $^{34}$ aplicaron el instrumento de Larson y observaron un nivel positivo de satisfacción, atribuyeron el puntaje positivo al reconocimiento de los pacientes como personas y a la habilidad técnica en el manejo de equipos y técnicas.

Valentine, Darby y Bonsel, ${ }^{35}$ en una encuesta diseñada para valorar la calidad del cuidado clínico y no clínico realizada en población general de 41 países con el fin de obtener criterios de calidad para el

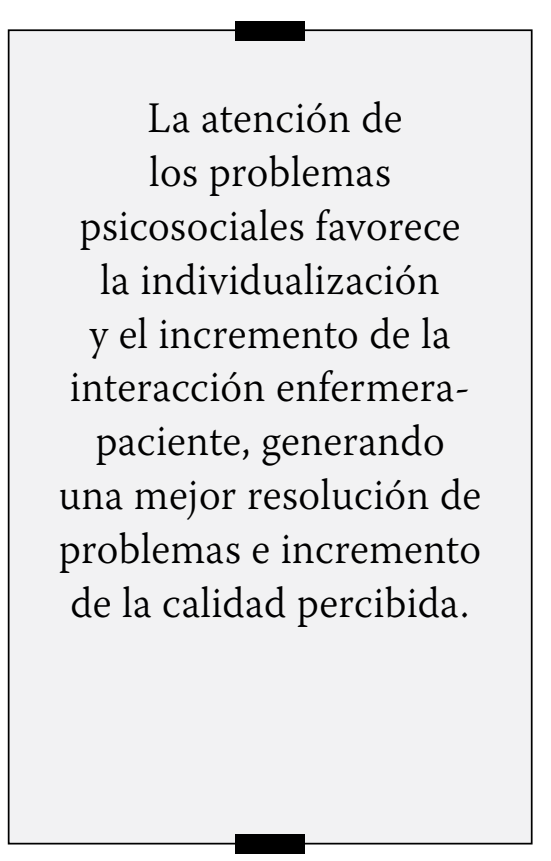

proceso de reforma política de la OMS, encontraron que respecto a la calidad del cuidado no clínico, la pronta respuesta, el reconocimiento de la dignidad y la comunicación interpersonal fueron factores ampliamente reconocidos por los encuestados.

Contreras, Flórez y Herrera ${ }^{36}$ validaron un instrumento de 72 reactivos para evaluar la adherencia terapéutica considerando factores socioeconómicos, factores relacionados con el proveedor y con las condiciones de salud del paciente, y factores relacionados con la terapia; entre los factores relacionados con el proveedor se consideró la actitud, la disponibilidad y la calidad de la comunicación, encontraron que las buenas relaciones entre los prestadores de los servicios de salud y los pacientes son imperativas para una buena adherencia.

$\mathrm{Al}$ año siguiente, Flórez ${ }^{37}$ estudió la adherencia terapéutica en 293 pacientes con factores de riesgo cardiovascular en un hospital de tercer nivel en Colombia y encontró que las relaciones entre enfermeras y pacientes influyen en la adhesión, además las percepciones de la enfermera y el paciente influyen en el proceso de interacción.

Para terminar con este eje de estudio, se menciona que Teng, Dai, Lotus, Wong, Chu y Tsai ${ }^{38}$ examinaron la influencia del compromiso profesional de las enfermeras sobre la seguridad del paciente y la percepción de la calidad del cuidado a través de un estudio transversal que involucró a 284 pares de enfermeras y pacientes, entre los hallazgos registrados encontraron que la empatía fue un factor importante en cuanto a la calidad percibida. Más de treinta años antes, LaMonica, 
Carew, Winder, Haase y Blanchard ${ }^{39}$ habían desarrollado un programa de relaciones humanas para el personal, observando los cambios favorables subsecuentes en la empatía del personal de enfermería.

Respecto al eje descrito, la mayoría de los estudios bajo diferentes modalidades consideran que la atención de los problemas psicosociales favorece la individualización y el incremento de la interacción enfermera-paciente, generando una mejor resolución de problemas e incremento de la calidad percibida. En otros casos se mostró correlación entre el componente de la interacción con la calidad del cuidado y con indicadores de eficiencia como disminución de los días de estancia, mejoría de datos clínicos y de la percepción del paciente.

En otras investigaciones se relacionó la percepción positiva del cuidado proporcionado por las enfermeras con la calidad del cuidado, pero se encontró que las variables sociodemográficas estaban incidiendo. Específicamente algunos estudios que mostraron incremento de la empatía, la relacionaron con la satisfacción del paciente $y$ con el aumento de calidad en general.

Una opinión muy generalizada, mostró que las relaciones interpersonales son uno de los indicadores de calidad más apreciados, sin embargo en el caso del eje descrito las limitaciones metodológicas de algunos estudios proponen la búsqueda de más evidencias en este rubro.

\section{Referencias Bibliográficas}

1. Bengoechea MB. Estudios sobre la comunicación paciente enfermera en cuidados intensivos. El estudio de las líneas de investigación pre- dominantes y otras relevantes. Enfermería Clínica 2001; 11(6): 266274.

2. Frich LMH. Nursing interventions for patients with chronic conditions. J Adv Nurs. 2003; 44(2): 137-153.

3. Finke EH, Light J \& Kitko L. A Systematic review of the effectiveness of nurse communication with patients with complex communication needs with a focus on the use of argumentative and alternative communication. J Clin Nurs. 2008; 17 (16): 2102-2115.

4. Tay LH. \& Hegney D. Factors affecting effective communication between registered nurses and cancer patients in an inpatient setting: a systematic review. Int J Evid Based Healthc. 2011; 9(2): 151-164.

5. Cossette S, Coté JK, Pepin J, Ricard $\mathrm{N} \&$ D'Aoust LJ. A dimensional structure of nurse-patient interactions from a care perspective: refinement of the Caring nursePatient Interaction Scale (CNPIShort Scale). J Adv Nurs. 2006; 55(2): 198-214.

6. Cossette S, Pepin J, Coté JK \& Courval FP. The multidimensionality of caring: a confirmatory factor analysis of the Caring Nurse Patient Interaction Short. J Adv Nurs. 2008; 61(6): 699-710.

7. Duffy JR, Hoskins L \& Furst S. Dimensions of caring. Psychometric Evaluation the Caring assessment tool. ANS Adv Nurs Sci. 2007; 30(3): 235-245.

8. Marriner A. y Raile M. Modelos y Teorías de Enfermería 6a ed España: Elsevier Mosby; 2008. p. 50116.

9. Watson J. Nursing: Human science and human care. Norwalk, CT: Appleton-Century-Crofts; 1985. p. 50-51.
10. Duffy JR. Caring Assessment Tools and the CAT-Admin. Chapter 12. En: Watson J. Assessing and Measuring Caring in Nursing and Health Science. New York USA: Springer Publishing Company; 2009. Pp. 131-141.

11. Watson J. Assessing and Measuring Caring in Nursing and Health Science. New York USA: Springer Publishing Company; 2009. p. 3-10, 131-141.

12. Medina JL. La Pedagogía del Cuidado: saberes y prácticas en la formación universitaria en Enfermería. Barcelona, España: Laertes, 1999. pp. 307.

13. Peplau H. Relaciones Interpersonales en Enfermería. Barcelona: Salvat Editores S.A.; 1990. p. 3-35.

14. Nightingale F. Notas sobre Enfermería. Qué es y qué no es. México: Salvat Editores; 1991. Pág. 139.

15. Henderson V. La Naturaleza de la Enfermería. Reflexiones 25 años después. Madrid, España: McGraw-Hill Interamericana; 1994. p. 2-37.

16. Pytel C, Fielden N, Meyer K \& Albert N. Nurse-patient/visitor Communication in the Emergency Department. J Emerg Nurs. 2009; 35(5): 406-411.

17. Beltrán-Salazar O. La práctica de enfermería en cuidado intensivo. Aquichán [Internet] 2008; [acceso el 15 de marzo de 2011]; 8(1): [50-63] Disponible en: http://bit. ly/Kth21K

18. Williams AM \& Irurita VF. Therapeutic an non-therapeutic interpersonal interactions: the patients perspective. J Clin Nurs. 2004; 3(7): 806-815.

19 Williams AM \& Kristianson LJ. Emotional care experienced by hospitalized patients: development and testing of a measure- 
ment instrument. J Clin Nurs. 2009; 18 (7): 1069-1077.

20. McCabe C. Nurse-patient communication: an exploration of patients' experiences. J Clin Nurs. 2004; 13 (1): 41-49.

21. Leeuwen R, Tiesinga LJ, Post D \& Jochemsen H. Spiritual care: implications of nurses professional responsibility. J Clin Nurs. 2006; 15(7):875-884.

22. Beltrán-Salazar O. La experiencia de estar hospitalizado en una unidad de cuidado intensivo. Aquichán. 2009; 9(1): 1-16.

23. Quintero MT y Gómez M. El cuidado de enfermería significa ayuda. Aquichán. 2010; 10 (1): 1-12.

24. Wysong PR \& Diver E. Patient's 'Perceptions of nurses' skill. Critical Care Nurse. 2009; 29(4): 24-37.

25. Kruijver IP, Garssen B, Visser AP \& Kuipper AJ. Signalizing psychosocial programs in cancer care: the structural use of short psychosocial checklist during medical or nursing visits. Patient Educ Couns. 2006; 62 (2): 163-177.

26. Charlton CR, Dearing KS, Berr JA \& Johnson MJ. Nurse practitioners 'communication styles and impact on patient outcomes: An integrated literature review. J Am Acad Nurse Pract. 2008; 20(7): 382-388.

27. Tapia-Valero C, Novo-García C, Hernández R, Sánchez B y Duarte $\mathrm{G}$. Las enfermeras mejoran la salud de los pacientes crónicos. Revisión sistemática. Evidentia. [Internet]. 2009; [Acceso el 11 de marzo de 2010]; 6(28): Disponible en: http://bit.ly/KR6Lf3

28. Thomas LH, McColl E, Priest J, Bond S. \& Boys R.J. Newcastle Satisfaction with Nursing Scales: an instrument for quality assessments of nursing care. Qual Health Care.
1996; 5:(2) 67-72.

29. Torres Contreras CC. Percepción de la calidad del cuidado de enfermería en pacientes hospitalizados. Avances en Enfermería. 2010; 28(2): 98-110.

30. De los Ríos JL. \& Sánchez-Sosa J. J. Well-being and medical recovery in the critical care unit: the role of the nurse-patient interaction. Salud Ment (Mex). 2002; 25(2): 2131.

31. Mok E. \& Chiu PC. Nurse-patient relationship in palliative care. J Adv Nurs. 2004; 48(5): 475-483.

32. Hojat M, Gonnella JS, Nasca TJ, Mangione S, Vergare M. \& Magee M. Physician empathy: definition, components, measurement, and relationship to gender and specialty. Am J Psychiatry. 2002; 159 (9): 1563-1569.

33. Miaofen Y, \& Li-Hua LA. Model for Testing the Relationship of Nursing Care and Patient Outcomes, Nurs Econ. 2004; 22(2): 75-80.

34. Sierra MT, Cardona LP, Bernal MF y Forero AE. Percepción del cuidado de Enfermería en diferentes instituciones hospitalarias de la ciudad de Bogotá. Avances en Enfermería. 2006; 24(1): 68-72.

35. Valentine N, Darby Ch. \& Bonsel, GJ. Which aspects of non clinical quality of care are most important? Results from WHO's general population surveys of Health Systems Responsiveness in 41 countries. Soc Sci Med. 2008; 66(9): 139-150.

36. Contreras A, Flórez IE, y Herrera A. Un instrumento para evaluar la adherencia: su validez facial y confiabilidad. Avances en Enfermería. 2008; 16 (2): 35-42.

37. Flórez IE, Adherencia a tratamientos en pacientes con factores de riesgo cardiovascular Avances en Enfermería [Internet]. 2009; [Acceso 10 de febrero de 2010]; 27(2): Disponible en: http://bit.ly/ IQ4we8

38. Teng CI, Dai YT, Lotus S, Wong MK, Chu TL \& Tsai YH. Professional Commitment, patient safety, and patient-perceived Care Quality. J Nurs Scholarsh. 2009; 41(3): 301-309.

39. LaMonica E, Carew D, Winder A, Haase AMB \& Blanchard K. Empathy training as the mayor thrust of a staff development program. Nurs Res. 1976; 25(6): 447-451.

\section{Agradecimientos}

A las Maestras Victoria Fernández García y Mercedes García Cardona, y a la Doctora Marina Menez Díaz por su apoyo para la búsqueda y adquisición de algunos artículos referidos; a los Doctores Marina Menez Díaz, Rebeca Robles García, Samuel Jurado Cárdenas y Francisco Juárez García por su apoyo en cuanto a la revisión de manuscritos previos, considerando sus recomendaciones para el logro de este artículo 